\title{
Allelic Diversity at the Merozoite Surface Protein-1 (MSP-1) Locus in Natural Plasmodium falciparum Populations: a Brief Overview
}

\author{
Marcelo U Ferreira ${ }^{+}$, Osamu Kaneko*, Masatsugu Kimura**, Qing Liu***, \\ Fumihiko Kawamoto***, Kazuyuki Tanabe ${ }^{* * * *}$
}

Departamento de Parasitologia, ICB, Universidade de São Paulo, Av. Prof. Lineu Prestes 1374, 05508-900 São Paulo, SP, Brasil *Department of Medical Zoology **Laboratory of Biophysics, Osaka City University Medical School, Osaka, Japan ***Department of International Health, Nagoya University School of Medicine, Nagoya, Japan ****Laboratory of Biology, Osaka Institute of Technology, Osaka, Japan

The merozoite surface protein-1 (MSP-1) locus of Plasmodium falciparum codes for a major asexual blood-stage antigen currently proposed as a major malaria vaccine candidate. The protein, however, shows extensive polymorphism, which may compromise its use in sub-unit vaccines. Here we compare the patterns of allelic diversity at the MSP-1 locus in wild isolates from three epidemiologically distinct malaria-endemic areas: the hypoendemic southwestern Brazilian Amazon $(n=54)$, the mesoendemic southern Vietnam $(n=238)$ and the holoendemic northern Tanzania $(n=79)$. Fragments of the variable blocks 2, 4a, $4 b$ and 6 or 10 of this single-copy gene were amplified by the polymerase chain reaction, and 24 MSP-1 gene types were defined as unique combinations of allelic types in each variable block. Ten different MSP-1 types were identified in Brazil, 23 in Vietnam and 13 in Tanzania. The proportion of genetically mixed infections (isolates with parasites carrying more than one MSP-1 version) ranged from 39\% in Brazil to 44\% in Vietnam and 60\% in Tanzania. The vast majority (90\%) of the typed parasite populations from Brazil and Tanzania belonged to the same seven most frequent MSP-1 gene types. In contrast, these seven gene types corresponded to only $61 \%$ of the typed parasite populations from Vietnam. Non-random associations were found between allelic types in blocks $4 a$ and 6 among Vietnamese isolates, the same pattern being observed in independent studies performed in 1994, 1995 and 1996. These results suggest that MSP-1 is under selective pressure in the local parasite population. Nevertheless, the finding that similar MSP-1 type frequencies were found in 1994 and 1996 argues against the prominence of short-term frequency-dependent immune selection of MSP-1 polymorphisms. Non-random associations between MSP-1 allelic types, however, were not detected among isolates from Brazil and Tanzania. A preliminary analysis of the distribution of MSP-1 gene types per host among isolates from Tanzania, but not among those from Brazil and Vietnam, shows significant deviation from that expected under the null hypothesis of independent distribution of parasites carrying different gene types in the human hosts. Some epidemiological consequences of these findings are discussed.

Key words: Plasmodium falciparum - malaria - allelic diversity - merozoite surface protein-1 - population genetics - vaccine candidate

Supported by grants from the Ministry of Education, Science, Sports and Culture of Japan, Toyota Foundation, the Program for Malaria Control in the Amazon of the National Health Foundation (Brazilian Ministry of Health), Fundação de Amparo à Pesquisa do Estado de São Paulo, Brazil, and the UNDP/World Bank/World Health Organization Special Programme for Research and Training in Tropical Diseases. MUF was supported by a research student scholarship from the Ministry of Education, Science, Sports and Culture of Japan.

${ }^{+}$Corresponding author: Fax: +55-11-818.7417. E-mail: muferrei@usp.br

Received 15 June 1998

Accepted 30 July 1998
The polymorphic merozoite surface protein-1 (MSP-1) of Plasmodium falciparum is a major asexual blood-stage malaria vaccine candidate (Holder 1996). Comparisons of nucleotide sequences led to the identification of seven variable blocks in the gene, which are interspersed with five conserved and five semi-conserved blocks (Fig. 1). There are essentially two versions of each block, named after the representative isolates MAD20 and K1 (Tanabe et al. 1987). A major exception to this dimorphic rule is the variable block 2 , that has a third version originally described in the isolate RO33 (Certa et al. 1987). Most allelic diversity is generated by intragenic recombination between 
these representative sequences at the 5 ' end of the gene, within blocks 3, 4 and 5. Minor differences also exist between homologous versions of the same variable block, and nucleotide substitutions (most of which are dimorphic) occur in semi-conserved and conserved blocks (Tanabe et al. 1987).

Major MSP-1 gene types may be defined as unique combinations of: (a) one of three versions of block 2 (MAD20, K1 or RO33), (b) one of four possible versions of block 4 , because recombination within this region generates MAD20/K1 and K1/MAD20 hybrids in addition to the 'pure' allelic types MAD20 and K1 (Conway et al. 1991b, Kaneko et al. 1996), and (c) one of two versions (MAD20 or K1) of the segment between the variable blocks 6 and 16, that comprises about $60 \%$ of the gene. Recombination events have not been described in this portion of the gene (Tanabe et al. 1987, 1989, Peterson et al. 1988, Conway et al. 1991b, Jongwutiwes et al. 1991, Kaneko et al. $1996,1997)$. Therefore, the $24 M S P-1$ gene types shown in Table I may theoretically be observed in natural parasite populations (Kaneko et al. 1997).

The extent of allelic diversity in different malaria-endemic areas should be evaluated if the variable domains of MSP-1, that are highly immunogenic (Holder \& Riley 1996), are to be included in subunit malaria vaccines. A novel polymerase chain reaction (PCR)-based strategy was recently developed to group clinical isolates of $P$. falciparum into the $24 M S P-1$ gene types defined in Table I (Kaneko et al. 1997). This strategy has been successfully applied to type wild isolates from the mesoendemic southern Vietnam (Kaneko et al. 1997, Ferreira et al. 1998b), the hypoendemic Brazilian Amazon (Ferreira et al. 1998a), and the holoendemic Tanzania (Ferreira et al. 1998c). In this communication we analyze available data regarding complete $M S P-1$ typing of isolates from these three malaria-endemic areas.

\section{MATERIALS AND METHODS}

Table II summarizes basic information regarding typed $P$. falciparum isolates in each malariaendemic area. Genomic DNA was extracted directly from the blood of $P$. falciparum-infected patients, without previous in vitro cultivation of parasites. Locations of the oligonucleotide primers are shown in Fig. 1. Primer sequences and PCR protocols are given elsewhere (Kaneko et al. 1997). The basic PCR-based typing procedure developed by Kaneko et al. (1997) may be described as it follows:

First step - Block 2 was typed in three separate reactions with the allelic specific forward primers $\mathrm{M} 2 \mathrm{~F}, \mathrm{~K} 2 \mathrm{~F}$ and R2F and the common reverse primer C3R.
TABLE I

Merozoite surface protein-1 (MSP-1) gene types defined as unique combinations of allelic types in each variable block

\begin{tabular}{|c|c|c|c|c|}
\hline \multirow[b]{2}{*}{ Gene type ${ }^{a}$} & \multicolumn{4}{|c|}{ Variable block } \\
\hline & 2 & $4 a$ & $4 \mathrm{~b}$ & 10 \\
\hline 1 & $\mathrm{~K} 1$ & $\mathrm{~K} 1$ & $\mathrm{~K} 1$ & $\mathrm{~K} 1$ \\
\hline 2 & MAD20 & $\mathrm{K} 1$ & $\mathrm{~K} 1$ & $\mathrm{~K} 1$ \\
\hline 3 & R033 & K1 & K1 & $\mathrm{K} 1$ \\
\hline 4 & $\mathrm{~K} 1$ & MAD20 & $\mathrm{K} 1$ & $\mathrm{~K} 1$ \\
\hline 5 & MAD20 & MAD20 & $\mathrm{K} 1$ & K1 \\
\hline 6 & RO33 & MAD20 & K1 & $\mathrm{K} 1$ \\
\hline 7 & K1 & K1 & MAD20 & K1 \\
\hline 8 & MAD20 & $\mathrm{K} 1$ & MAD20 & K1 \\
\hline 9 & RO33 & $\mathrm{K} 1$ & MAD20 & $\mathrm{K} 1$ \\
\hline 10 & K1 & MAD20 & MAD20 & K1 \\
\hline 11 & MAD20 & MAD20 & MAD20 & K1 \\
\hline 12 & RO33 & MAD20 & MAD20 & K1 \\
\hline 13 & K1 & $\mathrm{K} 1$ & K1 & MAD20 \\
\hline 14 & MAD20 & $\mathrm{K} 1$ & $\mathrm{~K} 1$ & MAD20 \\
\hline 15 & RO33 & $\mathrm{K} 1$ & $\mathrm{~K} 1$ & MAD20 \\
\hline 16 & $\mathrm{~K} 1$ & MAD20 & K1 & MAD20 \\
\hline 17 & MAD20 & MAD20 & K1 & MAD20 \\
\hline 18 & RO33 & MAD20 & K1 & MAD20 \\
\hline 19 & $\mathrm{~K} 1$ & $\mathrm{~K} 1$ & MAD20 & MAD20 \\
\hline 20 & MAD20 & K1 & MAD20 & MAD20 \\
\hline 21 & RO33 & K1 & MAD20 & MAD20 \\
\hline 22 & $\mathrm{~K} 1$ & MAD20 & MAD20 & MAD20 \\
\hline 23 & MAD20 & MAD20 & MAD20 & MAD20 \\
\hline 24 & RO33 & MAD20 & MAD20 & MAD20 \\
\hline
\end{tabular}

$a$ : each gene type is defined as a unique combination of allelic types detected in the variable blocks 2 , 4a (5' segment of block 4), 4b (3' segment of block 4) and 616 of the MSP-1 gene. Since there is no recombination at the central and C-terminal portions of this gene, the allelic type detected in block 10 is considered to be the same for the variable blocks 6, 8, 14 and 16. Allelic types are named after the reference isolates MAD20, K1 and RO33.

Second step - The gene fragments between the conserved block 5 and the variable block 6 were amplified in two separate reactions with the common forward primer $\mathrm{C} 5 \mathrm{~F}$ and the type-specific reverse primers M6R or K6R. Alternatively, block 10 was typed with the semi-conserved forward primer $\mathrm{C} 9 \mathrm{~F}$ and the type-specific reverse primers M10R and K10R. Since there is no recombination between blocks 6 and 16, the allelic type found in blocks 6 or 10 is the same for variable blocks 8,14 and 16.

Third step - Segments between blocks 2 and 6 were amplified in three separate reactions with the type-specific forward primers M2F, K2F or R2F, and the type-specific reverse primers M6R or K6R. The PCR fragments amplified in this step were used as template in the next step. As an alternative, this 
TABLE II

Recent polymerase chain reaction-based studies involving complete typing of the merozoite surface protein-1 gene in natural Plasmodium falciparum populations

\begin{tabular}{llcl}
\hline Area & $\begin{array}{l}\text { Malaria } \\
\text { endemicity }\end{array}$ & $\begin{array}{l}\text { No. of typed } \\
\text { isolates }\end{array}$ & Reference \\
\hline Brazilian Amazon & Low & 54 & Ferreira et al. 1998a \\
Southern Vietnam & Intermediate & 136 & Kaneko et al. 1997 \\
Southern Vietnam & Intermediate & 102 & Ferreira et al. 1998b \\
Northern Tanzania & High & 79 & Ferreira et al. 1998c \\
\hline
\end{tabular}

a

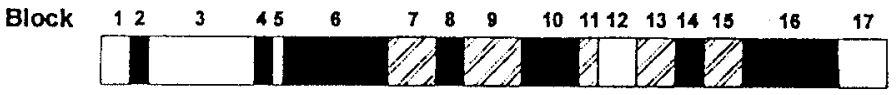

b

MAD20-type

K1-type

RO33-type
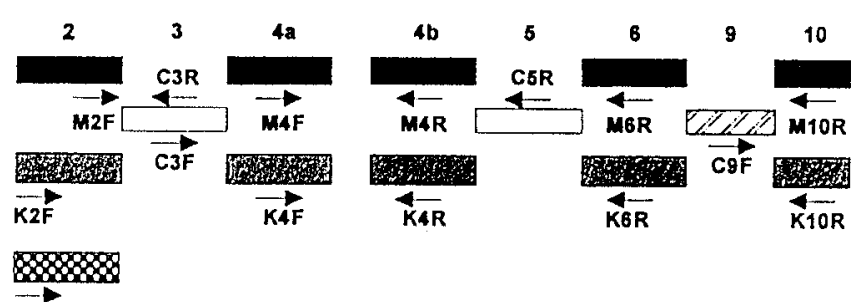

R2F

Fig. 1-a: structure of the merozoite surface protein-1 gene of Plasmodium falciparum. Conserved, semi-conserved and variable blocks of the gene are shown as open, hatched and closed boxes, respectively. Block numbers are after Tanabe et al. (1987); b: locations and directions of the oligonucleotide primers used to type blocks 2, 4a, 4b, 6, and 10 are also indicated. Redrawn from Kaneko et al. 1997.

template may be prepared with the conserved forward primer $\mathrm{C} 3 \mathrm{~F}$ and the conserved reverse primer C5R.

Forth step - Block 4 was typed by nested PCR in four separate reactions with the type-specific forward primers $\mathrm{M} 4 \mathrm{~F}$ or $\mathrm{K} 4 \mathrm{~F}$ and type-specific reverse primers M4R or K4R. As an alternative, the first step may be eliminated, and block 2 may be typed by detecting allelic-specific fragments in the second step (Ferreira et al. 1998b).

The detection of PCR products in the expected size ranges after $1.5-2 \%$ agarose gel electrophoresis defined the presence of each allelic type in blocks 2,6 or $10,4 \mathrm{a}$ and $4 \mathrm{~b}$. As MSP- 1 is a singlecopy gene in the haploid genome of blood-stage parasites, we consider that isolates harboring more than one gene type have mixed infections with genetically distinct $P$. falciparum subpopulations. Each subpopulation may be separately typed by this approach (Kaneko et al. 1997).

\section{RESULTS AND DISCUSSION}

Are all theoretically possible MSP-1 gene types found in natural Plasmodium falciparum populations? - As shown in Fig. 2, all but one of the 24 possible $M S P-1$ gene types were detected in mesoendemic Vietnam. Only gene type 11 was absent in that area at both occasions (Kaneko et al. 1997, Ferreira et al. 1998b). This suggests that almost all possible combinations of MSP-1 allelic types may be found in parasites that are able to infect human hosts. In contrast, only 10 and 13 $M S P-1$ types were found in hypoendemic Brazil and holoendemic Tanzania, respectively. Moreover, essentially the same $M S P-1$ gene types were found to predominate in both countries, and about $90 \%$ of the typed parasite populations belonged to the seven most common gene types, namely the types $13,16,17,18,22,23$ and 24 as defined in Table I (Ferreira et al. 1998c). Nevertheless, these seven gene types were found in only $61 \%$ of the typed parasite populations in Vietnam.

Is there any association between the extent of MSP-1 diversity and the intensity of malaria transmission? - If we compare the proportions of genetically mixed infections (that is, patients harboring more than one $M S P-1$ gene type) and the average number of $M S P-1$ gene types found per patient, an apparent positive association is found between malaria endemicity and $M S P-1$ diversity (Table III). However, if we compare the number of different $M S P-1$ gene types found in each en- 

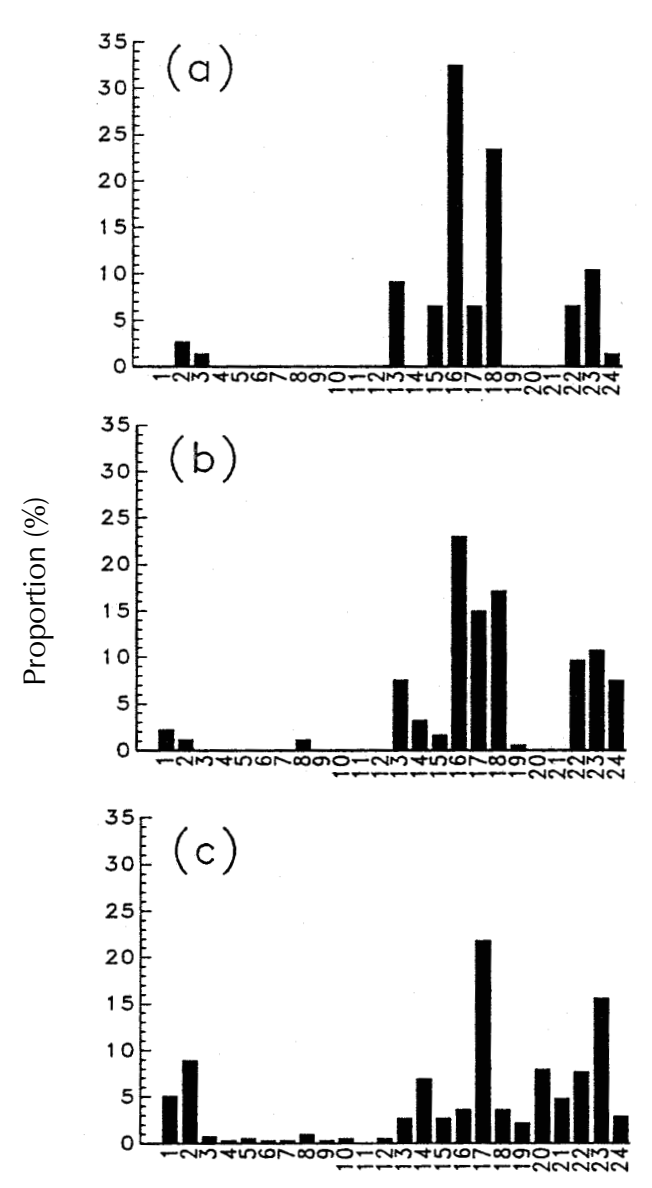

MSP-1 gene type

Fig. 2-a: frequency distribution of the merozoite surface protein-1 (MSP-1) gene types in 54 Plasmodium falciparum isolates collected in July 1995 in the city of Porto Velho, State of Rondônia, southwestern Brazilian Amazon (Ferreira et al. 1998a); b: frequency distribution of the MSP-1 gene types in 79 P. falciparum isolates collected between July and September 1996 in the city of Tanga and the nearby village of Pangani, in northern Tanzania (Ferreira et al. 1998c); c: frequency distribution of the $M S P-1$ gene types in 238 P. falciparum isolates collected between July 1994 and July 1996 from malaria patients belonging to the ethnic majority Kinh and the minority K'ho living in the towns of Bao Loc and Phu Rieng and nearby areas in southern Vietnam (Kaneko et al. 1997, Ferreira et al. 1998b). The 24 MSP-1 gene types are numbered as in Table I. demic area, no such association can be detected. Therefore, despite the fact that most infected hosts in Tanzania carry two or more parasite clones which may be ingested by the vector and recombine during meiosis, the resulting repertoire of MSP-1 variants seems to be relatively restricted in human hosts, if compared with the situation found in Vietnam. Strong selective pressure related to the sequential use of several different antimalarials in a few years, in the context of multi-drug resistance, may have resulted in increased genetic diversity of $P$. falciparum populations in Vietnam.

Are the patterns of MSP-1 diversity temporally stable in a given malaria-endemic area? - Fig. 3 compares the distribution of $M S P-1$ gene types in parasite populations sampled in the same communities in southern Vietnam at intervals of 12 months (Fig. 3a) and 18-24 months (Fig. 3b). There is no significant difference when both pairs of frequency distributions are compared. The stability in the frequencies of MSP-1 gene types over periods of 1224 months does not imply that long-term changes can be ruled out. Under the present conditions of malaria transmission in southern Vietnam, just a few infections with parasites carrying distinct versions of the MSP-1 antigen are expected per host at a one-year or two-year interval. As a consequence, natural acquisition of effective anti-MSP1 immunity may occur at a rather slow rate. We have now examined this issue in relation to the Brazilian Amazon by typing $M S P-1$ variable blocks in $P$. falciparum isolates collected over a period of 12 years (LA Silveira \& MU Ferreira, unpublished data).

Are there non-random associations between MSP-1 variable blocks in natural parasite populations? - If intragenic recombination occurs frequently at the MSP-1 locus in the absence of major selective constraints, the distribution of $M S P$ 1 gene types would be described by a simple probability model analogous to those used in population genetics to estimate expected frequencies of multiple-locus genotypes (Tibayrenc 1995). For instance, the expected frequency of gene type 1 (as defined in Table I) is given by multiplying the

TABLE III

The extent of allelic diversity at the merozoite surface protein-1 (MSP-1) locus in natural Plasmodium falciparum populations from areas with different levels of malaria endemicity

\begin{tabular}{llccc}
\hline Area & $\begin{array}{l}\text { Malaria } \\
\text { endemicity }\end{array}$ & $\begin{array}{l}\text { No. of } M S P-1 \\
\text { gene types } \\
\text { detected by PCR }\end{array}$ & $\begin{array}{l}\text { Proportion (\%) } \\
\text { of isolates with } \\
>1 M S P-1 \text { type }\end{array}$ & $\begin{array}{l}\text { Average no. of } \\
M S P-1 \text { types } \\
\text { per patient }\end{array}$ \\
\hline Brazil & Low & 10 & 39 & 1.42 \\
Vietnam & Intermediate & 23 & 44 & 1.76 \\
Tanzania & High & 13 & 60 & 2.37 \\
\hline
\end{tabular}


K’ho people 1994-95

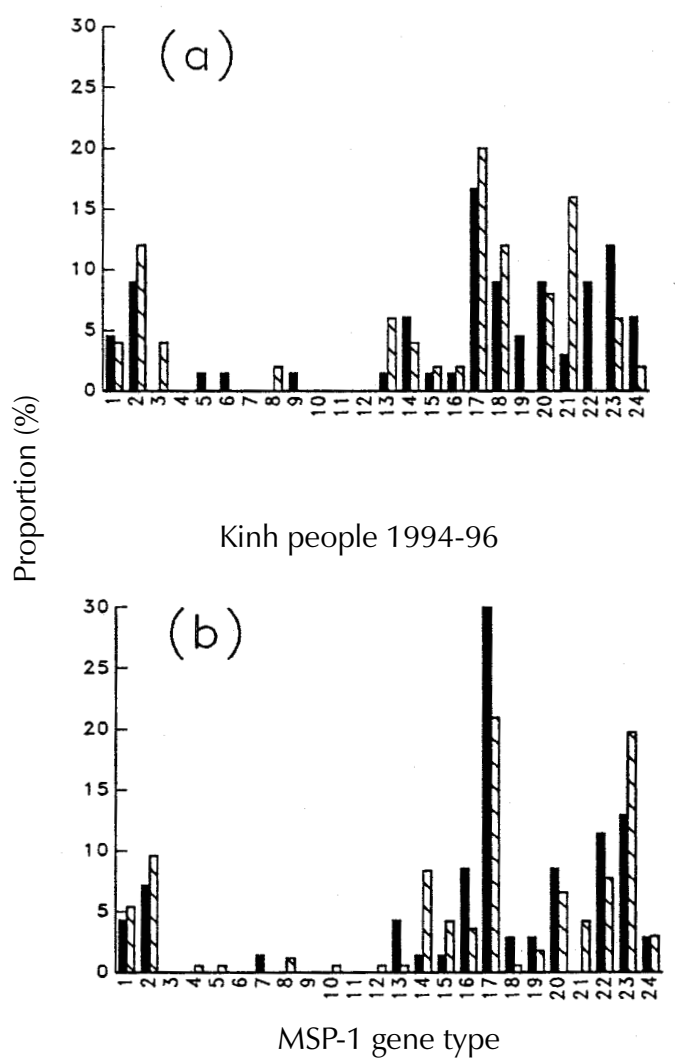

Fig. 3-a: frequency distribution of merozoite surface protein1(MSP-1) gene types in isolates from $\mathrm{K}$ 'ho people living in hill areas surrounding Bao Loc, southern Vietnam, collected between July-August $1994(n=34)$ (closed bars) and in August $1995(n=28)$ (striped bars) (redrawn from Kaneko et al. 1997); $\mathrm{b}$ : frequency distribution of $M S P-1$ gene types in isolates from Kinh people living in the town of Bao Loc, southern Vietnam, collected between July-August $1994(n=44)$ (closed bars) and between January-July $1996(n=95)$ (striped bars) (redrawn from Ferreira et al. 1998b).

observed frequencies of the allelic type $\mathrm{K} 1$ in blocks 2, 4a, 4b and 6-16 in a given population. Fig. 4 shows expected frequencies of $M S P-1$ gene types under the null hypothesis of random association of allelic types (MAD20, K1 or RO33) in each variable block in Brazil and Tanzania. No significant difference between expected and observed frequencies was detected by the $\mathrm{c}^{2}$ test for goodness of fit in both cases (Ferreira et al. 1998a, c). In contrast, significant differences between expected and observed frequencies of $M S P-1$ gene types were found in two surveys in southern Vietnam (Fig. 5). Non-random associations were found to occur, in both cases, between blocks $4 \mathrm{a}$ and 616: $M S P-1$ gene types with concordant allelic fami- lies (either MAD20 or K1) in blocks 4a and 6 or 10 were found more frequently than expected (Kaneko et al. 1997, Ferreira et al. 1998b). The reasons why similar results were not found in holoendemic Tanzania and hypoendemic Brazil remain to be elucidated.

Non-random associations between allelic types may result from: (a) geographic isolation leading to random genetic drift, (b) limited chances for intragenic recombination during meiosis in the mosquito vector due to the presence of few different $M S P-1$ versions and the low prevalence of mixed infections in human hosts, and (c) biological constrains which bias for particular associations. As

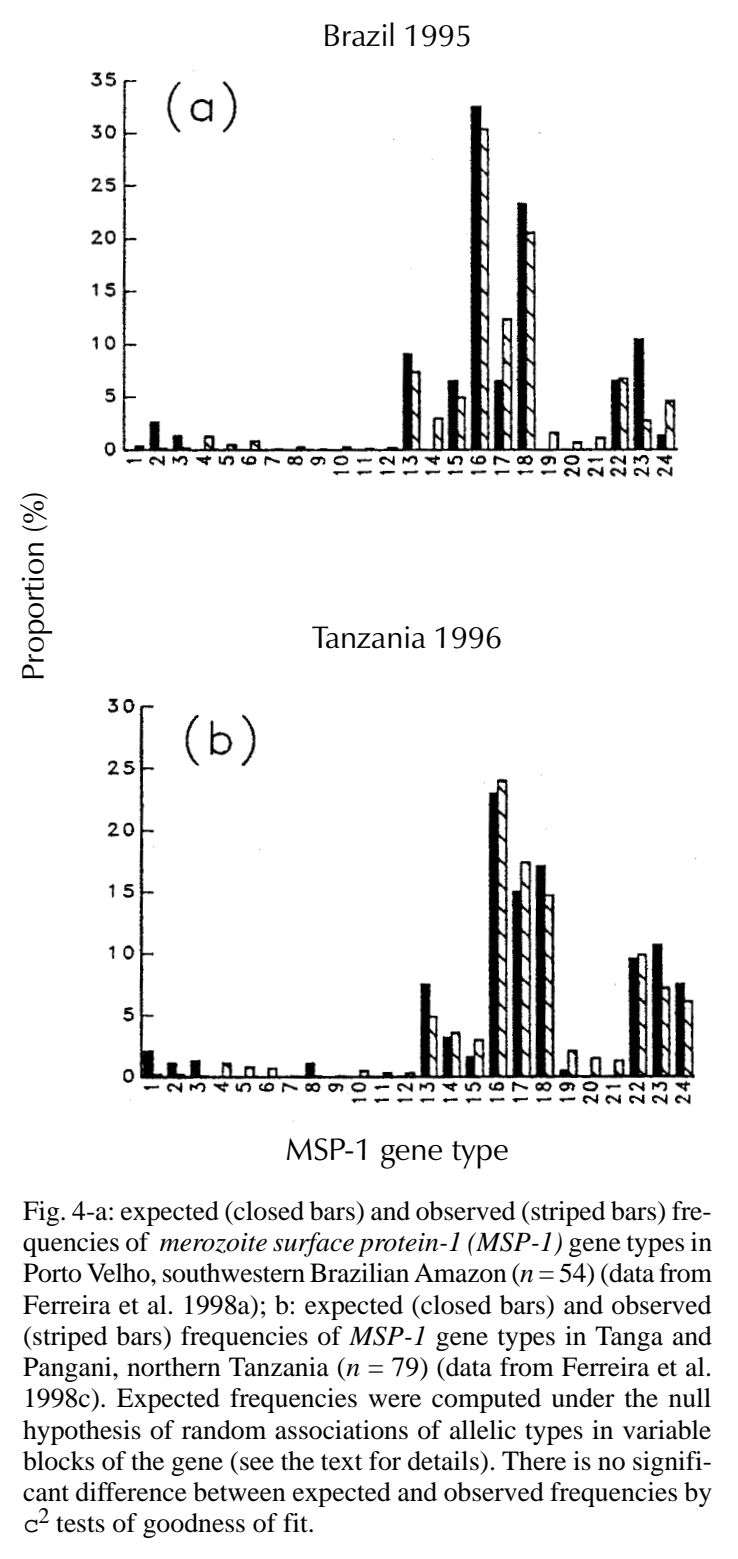


Vietnam 1994-95

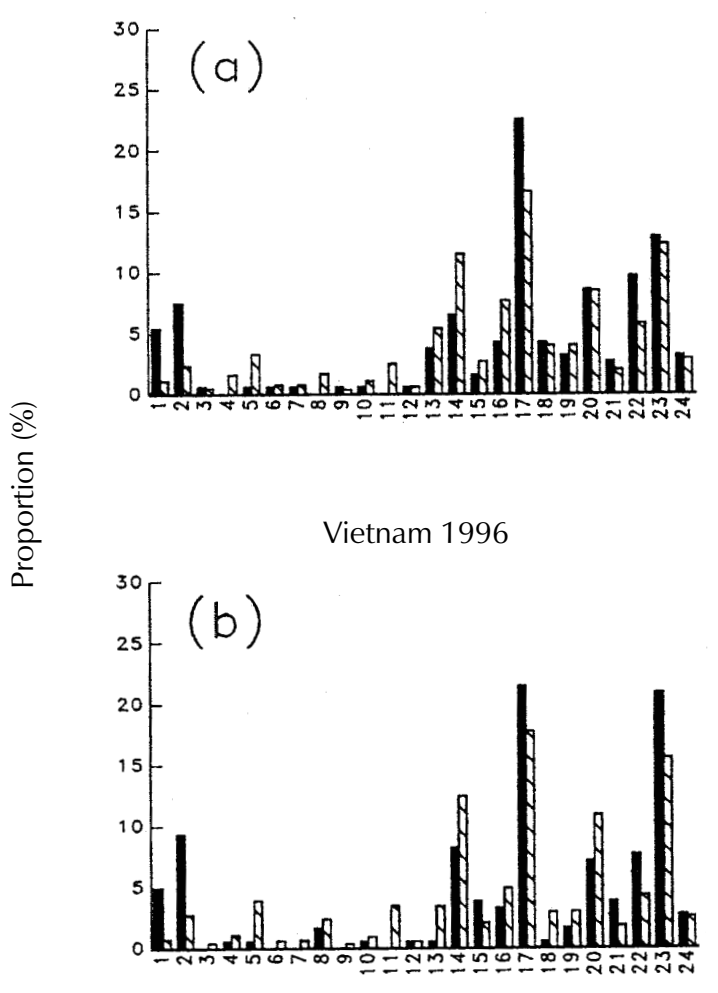

MSP-1 gene type
Fig. 5-a: expected (closed bars) and observed (striped bars) frequencies of merozoite surface protein-1 (MSP-1) gene types in isolates from Bao Loc, Vietnam, collected between July-August 1994 from both Kinh and K'ho people $(n=108)$ (data from Kaneko et al. 1997); b: expected (closed bars) and observed (striped bars) frequencies of $M S P-1$ gene types in isolates from Bao Loc, Vietnam, collected between January-July 1996 from both K'ho and Kinh people $(n=102)$ (data from Ferreira et al. 1998b). Expected frequencies were computed under the null hypothesis of random associations of allelic types in variable blocks of the gene (see the text for details). In both cases significant differences between expected and observed frequencies were detected by $c^{2}$ tests of goodness of fit. discussed elsewhere, the first two possible explanations do not match available data from Vietnam, and speculations regarding the third hypothesis are limited by the fact that the function of $M S P-1$ remains unknown (Ferreira et al. 1998b).

Are parasite populations carrying different MSP-1 gene types independently distributed in the host population? - A basic assumption of recent mathematical models of malaria transmission is that infections by different 'strains' are independent. This means that, in genetically mixed infections, a patient infected by a parasite carrying a given $M S P$ 1 gene type (for instance type 1 ) is as likely to be co-infected with a given second type (for instance type 2) as someone infected with any other MSP-1 type. This does not take into account the possibilities of: (a) frequent multiple-clone infections by vectors carrying two or more gene types including recombinant gene types resulting from the union of two different clones from one previous host (Hill \& Babiker 1995), and (b) either facilitation or competition between parasites carrying different versions of a polymorphic antigen which co-infect the same host (Gilbert et al. 1998). We applied here a simple statistical analysis to test this assumption.

The expected distribution of $M S P-1$ gene types per host under the hypothesis of independent distribution of $M S P-1$ gene types may be described as the sum of $N$ independent binomial distributions, where $N$ is the number of different $M S P-1$ gene types observed in host population. The variance of this summed binomial distribution, or expected variance $s^{2}$, was calculated and compared to the observed variance $s^{2}$ using a $c^{2}$ test as described (Lotz \& Font 1991). The difference between the expected and observed variances was statistically significant in Tanzania (Table IV), suggesting that the MSP- 1 gene types are not independently distributed in the host population. Therefore, we tested 21 possible pairwise associations between gene types in $2^{\prime} 2$ contingency tables using either standard $c^{2}$ or Fisher's exact tests when appropriate, with the significance level adjusted for multiple

\section{TABLE IV}

Statistical comparison of expected $\left(\mathrm{s}^{2}\right)$ and observed $\left(s^{2}\right)$ variances of the distribution of merozoite surface protein-1 (MSP-1) gene types among human hosts living in areas with different levels of malaria endemicity

\begin{tabular}{|c|c|c|c|c|c|}
\hline Area & $\begin{array}{l}\text { Malaria } \\
\text { endemicity }\end{array}$ & $\begin{array}{l}\text { Expected } \\
\text { variance }\left(s^{2}\right)\end{array}$ & $\begin{array}{l}\text { Observed } \\
\text { variance }\left(s^{2}\right)\end{array}$ & $\begin{array}{l}\mathrm{c}^{2} \\
(\mathrm{~d} . \mathrm{f} .)^{a}\end{array}$ & $P$ \\
\hline Brazil & Low & 1.04 & 0.32 & $16.45(53)$ & $>0.05$ \\
\hline Vietnam & Intermediate & 1.35 & 1.42 & $105.65(101)$ & $>0.05$ \\
\hline Tanzania & High & 1.59 & 2.10 & $103.08(78)$ & $<0.05$ \\
\hline
\end{tabular}

$a$ : degrees of freedom. 
comparisons with the Bonferroni's correction (Lord et al. 1997). At least 1 pair of $M S P-1$ gene types (18 and 24) was found to be positively associated $\left(P=0.0008\right.$ by $c^{2}$ test $)$. With the Bonferroni's correction applied to these data, an association is statistically significant at the 5\% level if $P<0.0024$. This means that the genetically similar types 18 and 24 , that differ only in the block $4 \mathrm{~b}$ allelic type (Table I), tend to co-occur more frequently than expected under the null hypotheses that they are independently transmitted. Nevertheless, departures from the null hypothesis of independent transmission were not detected in areas of lower endemicity such as Brazil and Vietnam (Table IV).

Mathematical models have recently regarded malaria as a heterogeneous disease caused by several independently transmitted and antigenically distinct parasite subpopulations or 'strains' that do not interact within the human hosts and are able to elicit 'strain'-specific protective immunity. These models estimate the basic reproduction number $R_{0}$ of malaria, defined as the average number of secondary infections generated by one primary infection in a fully susceptible population, as a weighted average of $R_{0}$ values for each 'strain'. This estimate is substantially lower than $R_{0}$ values obtained by conventional methods, suggesting that malaria eradication in Africa may be quite feasible (Gupta et al. 1994). Nevertheless, the finding that genetically and antigenically distinct parasite populations are not independently distributed in the human hosts in areas of high endemicity, such as northern Tanzania (Ferreira et al. 1998c) and the Gambia (Conway et al. 1991a), implies $R_{0}$ values considerably higher than those provided by the weighted average approach (Lord et al. 1997).

In conclusion, this study provides examples of the use of simple molecular and statistical approaches to investigate the extent of antigenic diversity in malaria parasites and to test hypotheses regarding the patterns of transmission and interaction of genetically distinct parasite subpopulations in endemic areas.

\section{ACKOWLEDGMENTS}

To HV Thien (Lam Dong II Provincial Hospital, Bao Loc, Vietnam), BT Ndawi (Primary Health Care Institute, Iringa, Tanzania), M Zhou and S Isomura (Nagoya University School of Medicine, Nagoya, Japan), AM Katzin and EAS Kimura (Department of Parasitology, University of São Paulo, Brazil).

\section{REFERENCES}

Certa U, Rotmann D, Matile H, Reber-Liske RA 1987. A naturally occuring gene encoding the major surface antigen precursor p190 of Plasmodium falciparum lacks tripeptide repeats. EMBO J 6: 4137-4142.
Conway DJ, Greenwood BM, McBride JS 1991a. The epidemiology of multiple-clone Plasmodium falciparum infections in Gambian patients. Parasitology 103: 1-6.

Conway DJ, Rosário V, Oduola AMJ, Salako AL, Greenwood BM, McBride JS 1991b. Plasmodium falciparum: intragenic recombination and nonrandom associations between polymorphic domains of the precursor to the major surface antigens. Exp Parasitol 73: 469-480.

Ferreira MU, Liu Q, Kaneko O, Kimura M, Tanabe K, Kimura EAS, Katzin AM, Isomura S, Kawamoto F 1998a. Allelic diversity at the merozoite surface protein-1 locus of Plasmodium falciparum in clinical isolates from the southwestern Brazilian Amazon. Am J Trop Med Hyg, in press.

Ferreira MU, Liu Q, Zhou M, Kaneko O, Kimura M, Thien HV, Isomura S, Tanabe K, Kawamoto F 1998 b. Stable patterns of allelic diversity at the merozoite surface protein-1 locus of Plasmodium falciparum in clinical isolates from southern Vietnam. J Euk Microbiol 45: 131-136.

Ferreira MU, Liu Q, Kimura M, Ndawi BT, Tanabe K, Kawamoto F 1998c. Allelic diversity in the merozoite surface protein-1 and epidemiology of multipleclone Plasmodium falciparum infections in northern Tanzania. J Parasitol, in press.

Gilbert SC, Plebanski M, Gupta S, Morris J, Cox M, Aidoo M, Kwiatkowski D, Greenwood BM, Whittle HC, Hill ASV 1998. Association of malaria parasite population structure, HLA, and immunological antagonism. Science 279: 1173-1177.

Gupta S, Trenholme K, Anderson RM, Day KP 1994. Antigenic diversity and transmission dynamics of Plasmodium falciparum. Science 263: 961-963.

Hill WG, Babiker HA 1995. Estimation of numbers of malaria clones in blood samples. Proc $R$ Soc London B 262: 249-257.

Holder AA 1996. Preventing merozoite invasion of erythrocytes, p. 77-104. In SL Hoffman, Malaria Vaccine Development. A Multi-immune Response Approach, ASM Press, Washington D.C.

Holder AA, Riley EM 1996. Human immune response to MSP-1. Parasitol Today 12: 173-174.

Jongwutiwes S, Tanabe K, Nakazawa S, Uemura H, Kanbara H 1991. Coexistence of gp195 alleles of Plasmodium falciparum in a small endemic area. Am J Trop Med Hyg 44: 299-305.

Kaneko O, Jongwutiwes S, Kimura M, Kanbara H, Ishii A, Tanabe K 1996. Plasmodium falciparum: variation in block 4 of the precursor to the major surface proteins (MSP1) in natural populations. Exp Parasitol 84: 92-95.

Kaneko O, Kimura M, Kawamoto F, Ferreira MU, Tanabe K 1997. Plasmodium falciparum: allelic variation in the merozoite surface protein 1 in wild isolates from southern Vietnam. Exp Parasitol 86: 45-57.

Lord CC, Woolhouse MEJ, Barnard BJH 1997. Transmission and distribution of virus serotypes: African horse sickness in zebra. Epidemiol Infect 118: 43-50. 
638 Genetic Diversity in Plasmodium falciparum - Marcelo U Ferreira et al.

Lotz JM, Font WF 1991. The role of positive and negative interspecific associations in the organization of communities of intestinal helminths of bats. Parasitology 103:127-138.

Peterson MG, Coppel RL, Moloney MB, Kemp DJ 1988. Third form of the precursor to the major surface antigens of Plasmodium falciparum. Mol Cell Biol 8: 2664-2667.

Tanabe K, Murakami K, Doi S 1989. Plasmodium falciparum: dimorphism of the p190 alleles. Exp Parasitol 68: 470-403.

Tanabe K, Mackay M, Goman M, Scaiffe JG 1987. Allelic dimorphism in a surface antigen gene of the malaria parasite Plasmodium falciparum. J Mol Biolol 195: 273-287.

Tibayrenc M 1995. Population genetics of parasitic protozoa and other microorganisms. Adv Parasitol 36: 47-115. 